This article has been scanned by iThenticat No plagiarism detected

Volume 3, Issue 2, April 2021

p. $18-27$

\title{
PROVIDING A SCENARIO OF WASTAGE MITIGATION IN THE HUMAN RESOURCE MANAGEMENT SYSTEM BASED ON KAIZEN THEORY IN THE MINISTRY OF EDUCATION OMAN
}

http://dx.doi.org/10.47832/2757-5403.2-3.3

\section{Ismail Bin Ali Bin Mohammed ALRAISI ${ }^{1}$, Rosni Bin SAMAH ${ }^{2} \&$ Azlan Bin Shaiful BAHARUM 3}

\begin{abstract}
:
his study aims to provide a scenario of waste mitigation in light of the Kaizen approach for Human Resources Development and Management Departments in the Ministry of Education (MOE)- Oman. It is also set to identify the manifestations and level of waste, from the point of view of the study sample, in these departments. In addition, it focuses on pointing out the differences in the level of waste in those relevant departments in accordance with gender, job level, academic qualification, years of experience, and department. This study proceeded, with the descriptive approach, to determine the manifestations and level of waste and used the scenario method to build the proposed scenario to reduce waste in light of the Kaizen approach to improve the organizational situation in these departments at MOE. The researcher has built an interview tool to form the proposed scenario and developed a questionnaire consisting of 30 items in three areas of waste, namely: Muda Mura and Muri. The questionnaire was utilized to spot the manifestations and level of waste, as well as the point of view of all directors and departments heads of the relevant departments regarding the impact of reducing waste in improving the organizational position of these departments. Moreover, 123 individuals were selected for the sample study. The study concluded that waste in Human Resources Development and Management Departments in the Ministry of Education was of a moderate degree. The results also revealed that there are no statistically significant differences at the level of $(a \leq 0.05)$ in the waste level of these departments due to the social variable in the study topics as a whole. The results showed that there are statistically significant differences between job titles levels in all topics of the study. The study concluded that a proposed scenario was developed to reduce waste in light of the Kaizen approach, including a number of

\footnotetext{
${ }^{1}$ Researcher, Islamic Science University of Malaysia, Nilai, ismail-alraisi@moe.om, https://orcid.org/0000-00024606-2704

${ }^{2}$ Supervisor, Prof. Dr. , Islamic Science University of Malaysia, Nilai, rosin@usim.edu.my

${ }^{3}$ Supervisor, Dr. , Islamic Science University of Malaysia, Nilai, azlan arab@usim.edu.my
}

Copyright (C) Published by IJHER Journal, www.ijherjournal.com Rimar Academy, Fatih, Istanbul, 34093 Turkey

All rights reserved 
requirements and components, for Human Resources Development and Management Departments in the Ministry of Education.

Key words: Kaizen Theory, Wastage, The Human Resource.

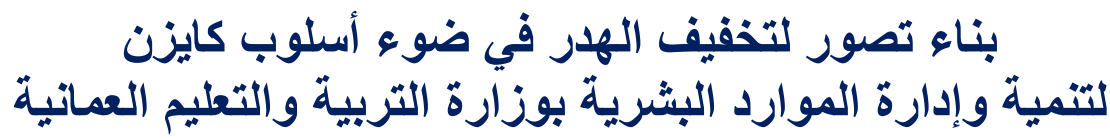

4

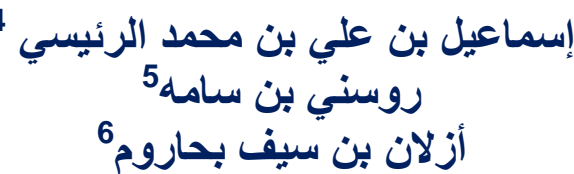

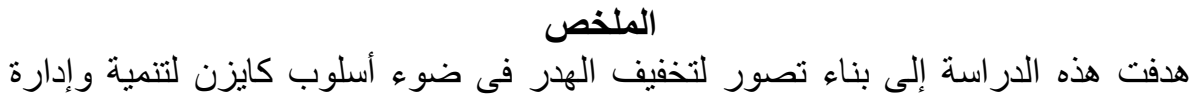

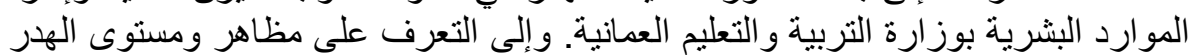

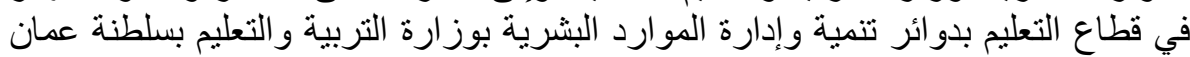

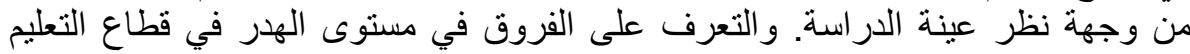

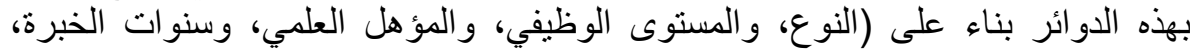

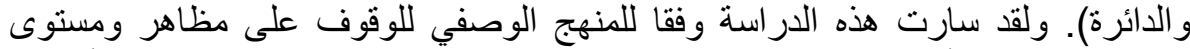

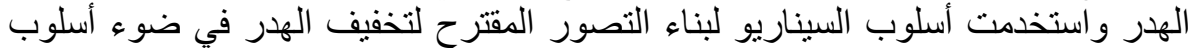

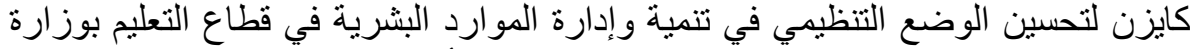

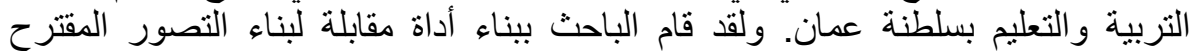

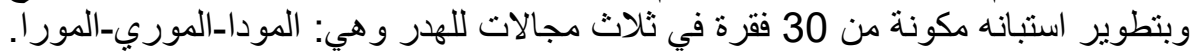

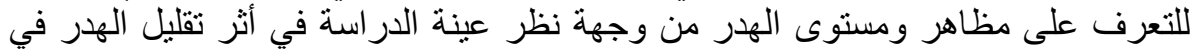

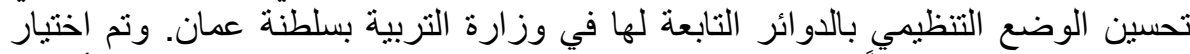

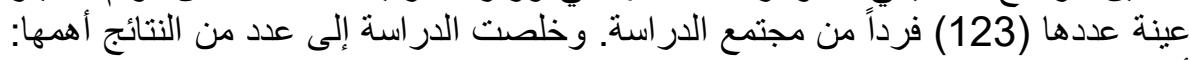

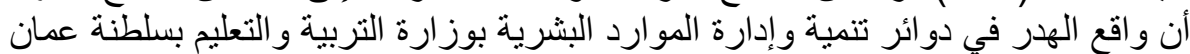

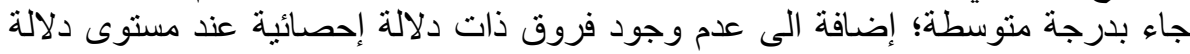

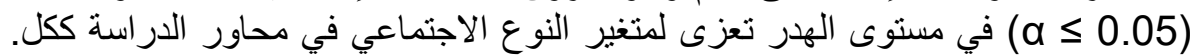

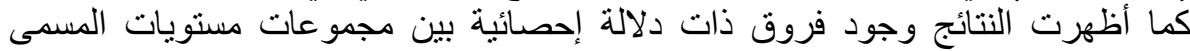

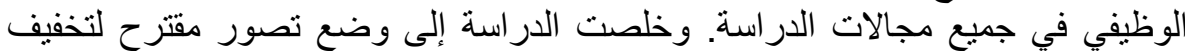

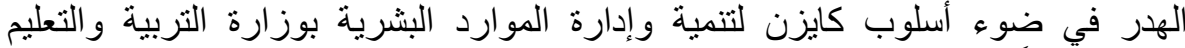

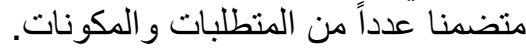
الكلمات المفتاحية: : الهدر، الموارد البشرية، أسلوب كايزن.

تسعى كل منظمة لتحقيق أهدافها من خلال إدارة مو اردها بما يتو ائم مع المستجدات المعاصرة، وبأحدث الأساليب المتبعة

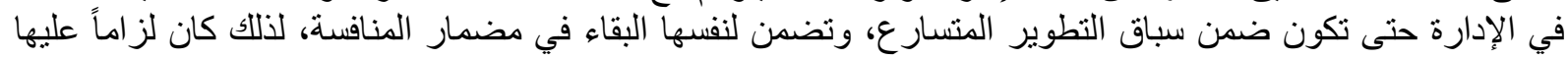

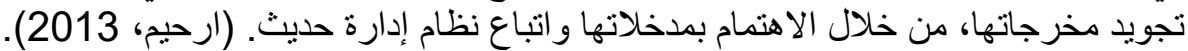

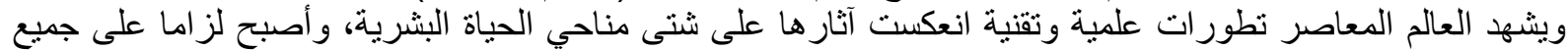

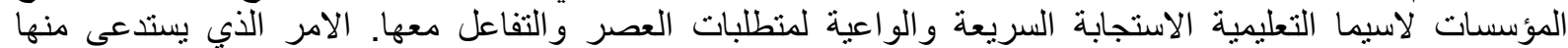
مر اجعة أدو اتها و آليات عملها لإثبات نجاحها في مواكبة التطور ات و المستجدات الحديثة، وذللك عن طريق إعادة النظر في 


\section{PROVIDING A SCENARIO OF WASTAGE MITIGATION IN THE HUMAN \\ RESOURCE MANAGEMENT SYSTEM BASED ON KAIZEN THEORY IN THE \\ MINISTRY OF EDUCATION OMAN}

أسلوب إدارتها للموارد، وأن تأخذ بعين الاعتبار التغيرات والتطور ات الحديثة لاستنباط أفضل الطرق والأساليب والعمل على استثمار ها بما يحقق أهدافها بارئ بفاعلية وكفاءة.

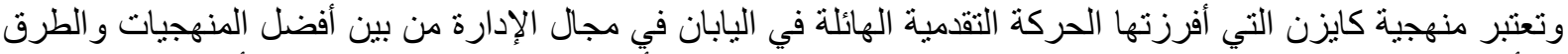

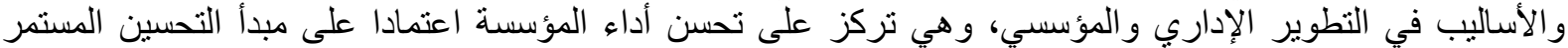

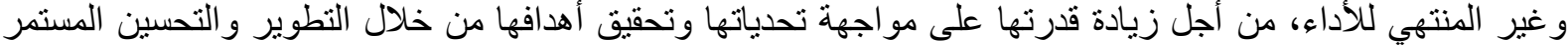

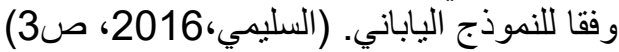
وذللك من خلال إدخال تحسينات مستمرة تدريجية بسيطة تساهم في تقليل التكاليف والقضاء على الهرر وزيادة الإنتاجية.

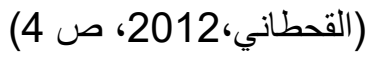

مشكلة الاراسة جاء اهتمام الباحث بمشكلة الدراسة كونه أحد العاملين بالمجال التربوي في وزارة التربية والتعليم وعلى دراية التهائ خاصة

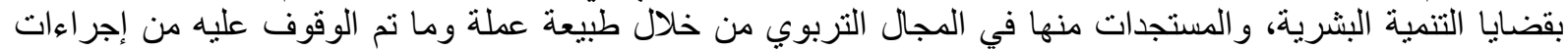

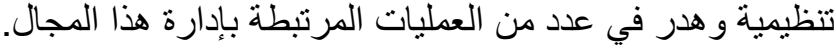

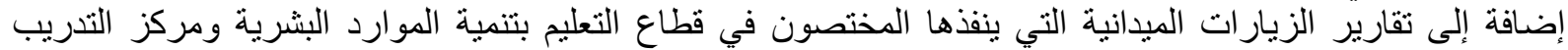

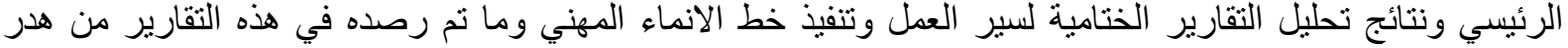
و عدم الالتز ام في تحقيق الاهداف المرسومة وإلى وجود تحديات في دئي دوائر تنمية الموارد البشرية بالمحافظات التعليمة و الحاجة إلى تطوير الوضع الترئ التنظيمي بها.

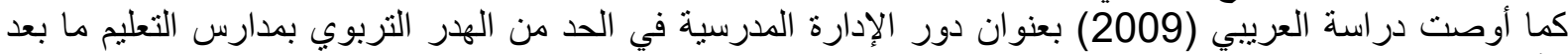

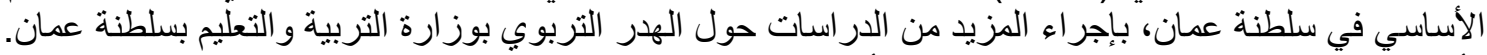

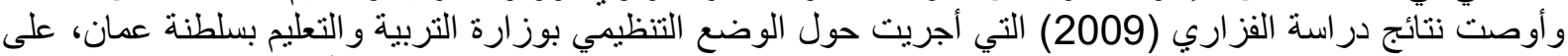
ضرورة تطوير الوضع التنظيمي لإدارة الموارد البشرية ومر اجعة التنظيم الحالي ومر اجعة الأهداف التئية المطلوبة من إدارة

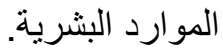
وشعر الباحث بأن بناء تصور لتخفيف الهدر في ضوء أسلوب كايزن لتتمية وإدارة الموارد البشرية بوزارة التربية

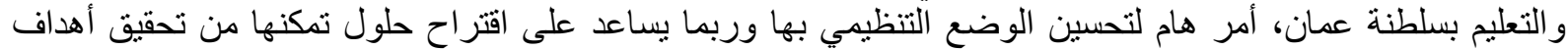

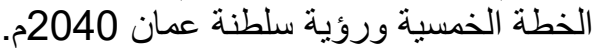

$$
\text { سعت هذه الداسة: }
$$

• التعرف على مظاهر ومسنوى الهار في دوائر تنمية وإدارة الموارد البشرية في قطاع التعليم بوزارة التربية والتعليم بسلطنة عمان.

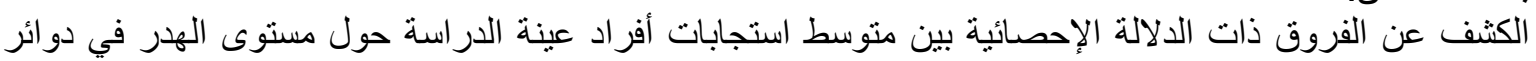

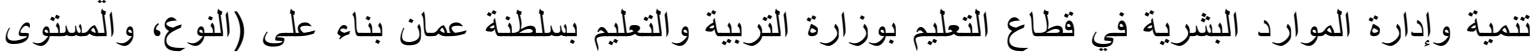

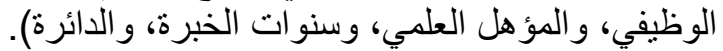
هناء تصور مقترح لتخفيف الهدر وتحسين الوضع التنظيمي لتنمية و إدارة الموارد البشرية في قطاع التربية بسلطنة عمان في ضوء أسلوب كايزن.

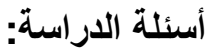

ما مظاهر ومستوى الهدر في دوائر تتمية وإدارة الموارد البشرية في قطاع التعليم بوزارة التربية والتعليم بسلطنة عمان؟

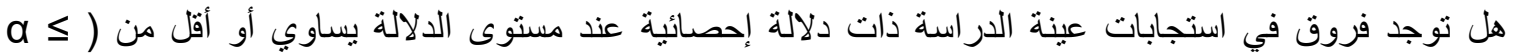

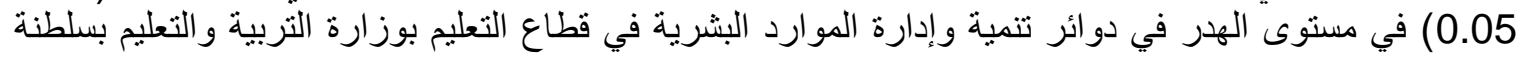

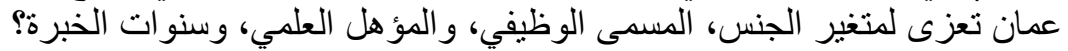

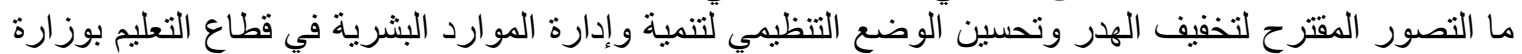
التربية و التعليم بسلطنة عمان في ضوء أسلوب كايزن؟ 


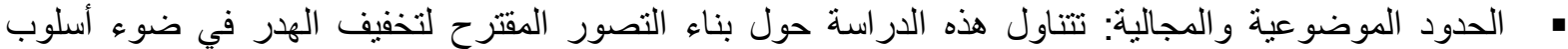

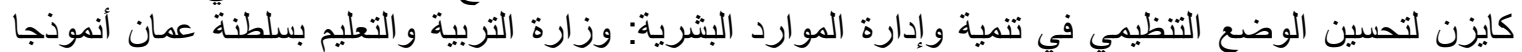

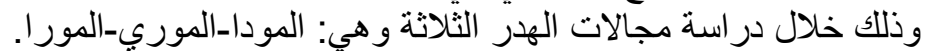

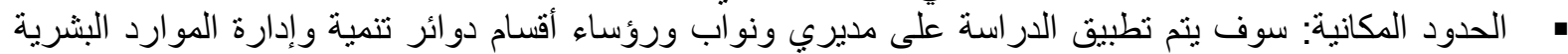

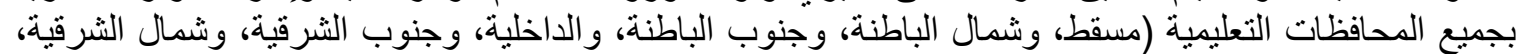

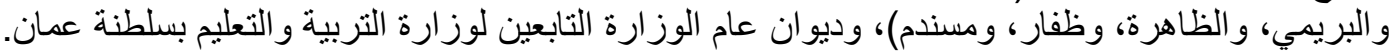
الحدود الزمنية: جري تطبيق الدراسة في الفصل الدراسي الثاني للعام الدراسي (2019/ 2020).

الإطار النظري والاراسات السابقة

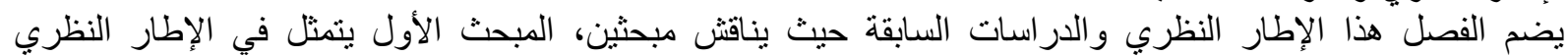

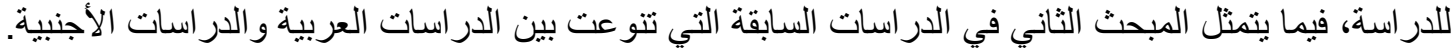

المبحث الأول: الإطار النظري للارباسة. تناول عرض الإطار النظري أربعة محاور رئيسية وهي: الوضع التنظيمي لإدارة وتتمية الموارد البشرية، النطوير

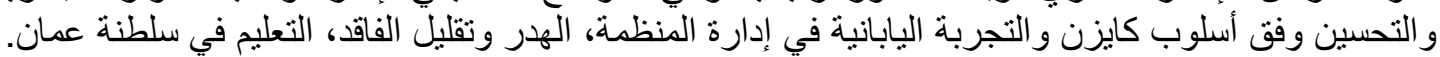

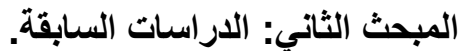

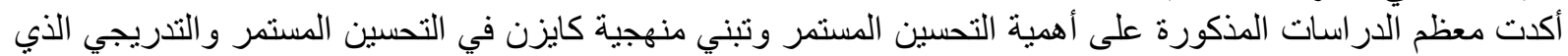
يعمل على رفع مستوى الأداء وزيادة الفاعلية، وتقليل الهدر في المؤسسة، ويمكن أن نستخلص من من هذه الدراستات النّات النقاط النالية: هناك توجه حديث و اهتمام متز ايد من قِبِل الباحثين بدراسة التحسين المستمر واعتماد أسلوب كايزن في تحقيق ذللك، من التِ

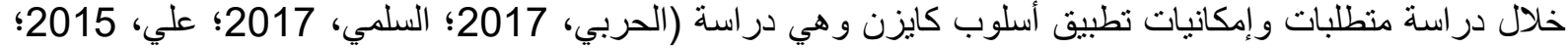

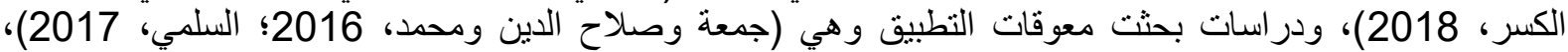

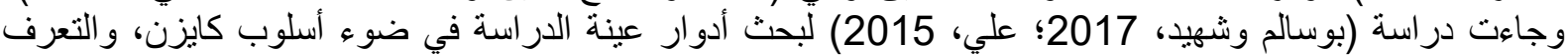

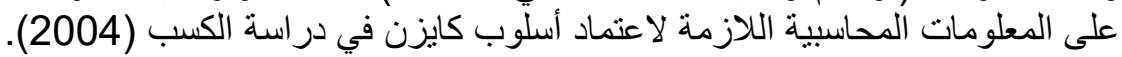

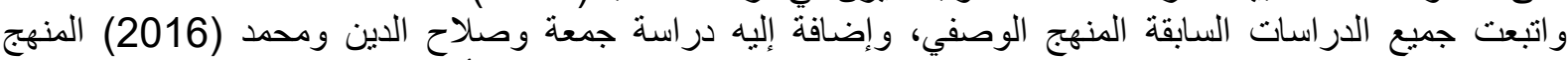

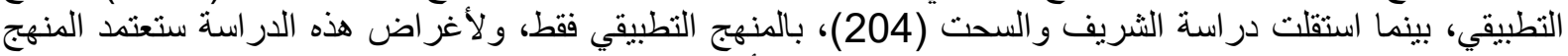

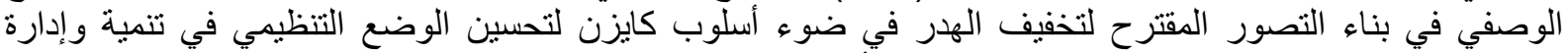
الموارد البشرية: وزاءرة التربية والتعليم بسلطنة عمان أنموذجا

\section{منهج الدراسة}

سارت هذه الدراسة وفقا للمنهج الوصفي القائم على جمع الحقائق و البيانات وتصنيفها ومعالجتها وتحليلها تحليلا دقيقا

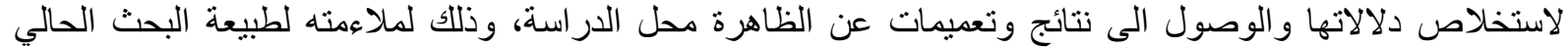

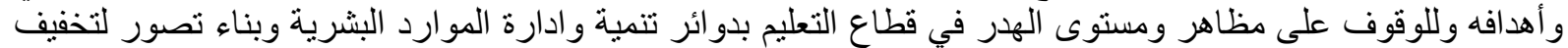

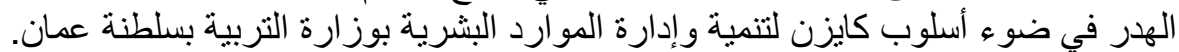

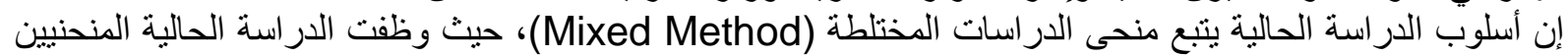

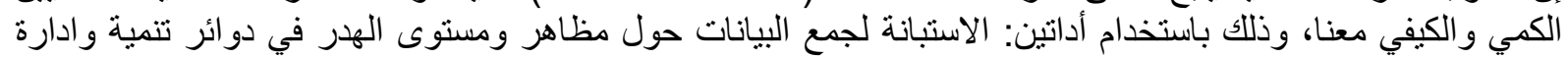

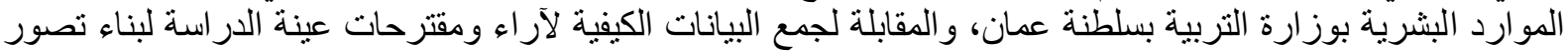

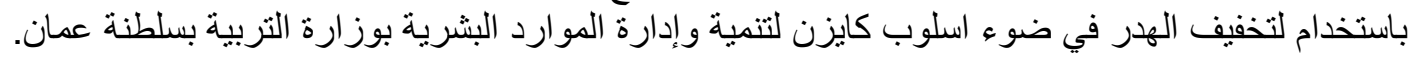

مجتمع الاراسة يتكون مجتمع الدراسة من جميع مديري ونو اب ورئ ورؤساء أقسام دو ائر تنمية و إدارة الموارد البشرية بوزارة التربية و التعليم بسلطنة عمان، والبالغ عددهم (180) في جميع المحافظات التعليمية بسلطنة عمان.

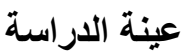
تكونت عينة الدر اسة التي طبقت عليها أداة الاستبانة من مديري ونو اب ورؤساء أقسام دوائر تنمية و إدارة الموارد البشرية

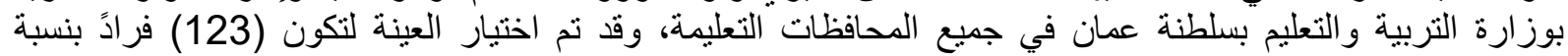
(68.34\%) من حجم المجتمع، ونم تحديدها بحسب المعايير الإحصائية التي نم تحديدها من قبل كرجسي ومروغان التاني (1970)، لهذا النوع من الدراسات ومجتمعها من الأفراد. 


\section{PROVIDING A SCENARIO OF WASTAGE MITIGATION IN THE HUMAN \\ RESOURCE MANAGEMENT SYSTEM BASED ON KAIZEN THEORY IN THE \\ MINISTRY OF EDUCATION OMAN}

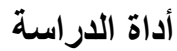

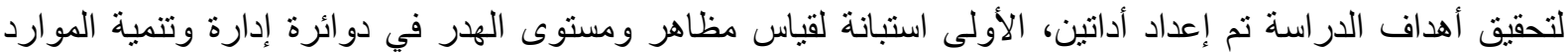

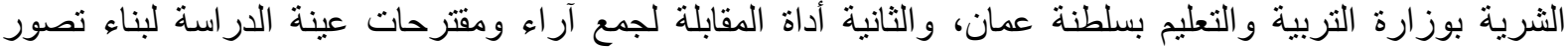

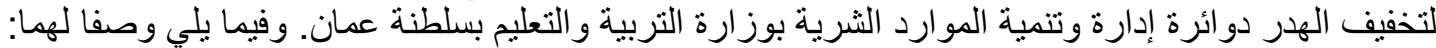

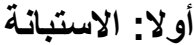

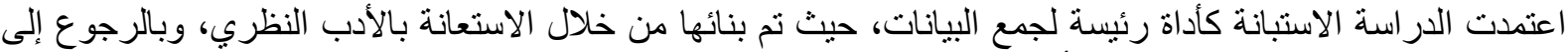

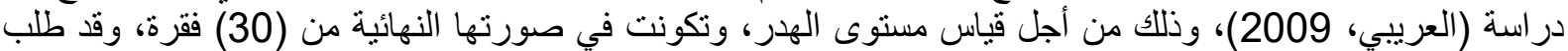

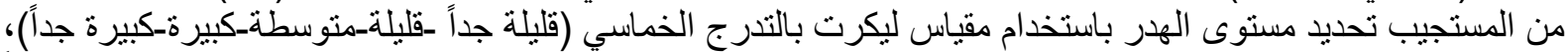

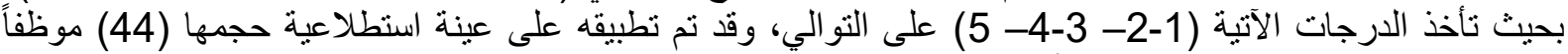

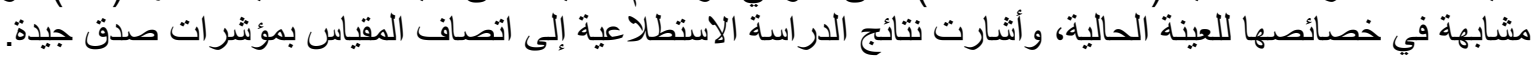

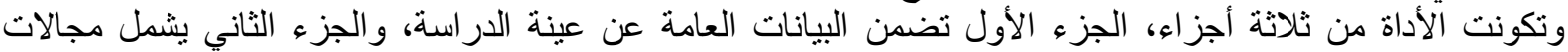

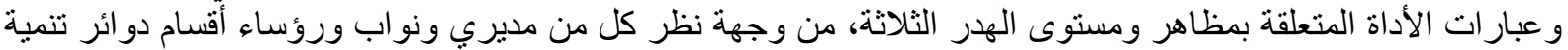
و إدارة الموارد البشرية، أما الجزء الثالث فيشمل المقترحات التي يطرحها المستجيب حول تقليل الهدر وتحسين الوضع

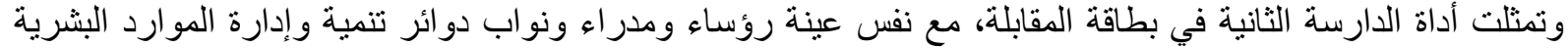

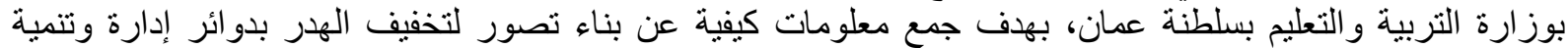
الموارد الثرية بوزارة التربية و التعليم بسلطنة عمان.

متغيرات الاراسة تكونت الدراسة من المتغير المنير ات الاتية:

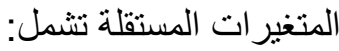

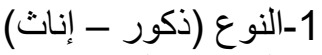
2-المستوى الوظيفي (مدير -نائب مدير - رئيس قسم)

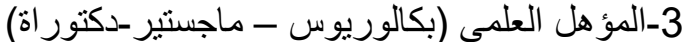

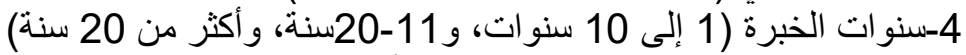
المتغير التابع: ويتمثل قياس مستوى الهدر بأنواعه (المودا-المور ا-الموري).

المعالجة الاحصائية

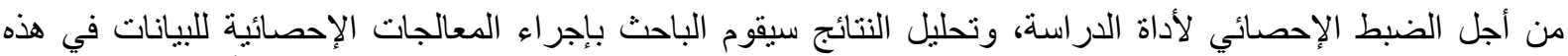

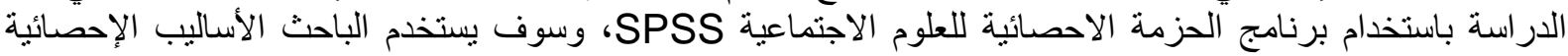

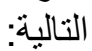
التكرارات، والنسب المئوية، والمتوسطات الحسابية، والانحر افات المعيارية، لقياس السؤوال الثاني من أسئلة الار اسة للوقوف على مستوى الهدر. اختبار "ت" لعينة واحدة لحساب دلالة الفروق بين متغيرات الدراسة المنمنلة في (النوع) وتحليل التباين الأحادي (One-Way ANOVA) الخبرة) • معامل ألفا كرونباخ، لحساب ثبات أدوات الدراسة ولتحديد الصدق الداخلي للأداة.

نتائج الاراسة وخلصت الدر اسة إلى عدد من النتائج أهمها: وجود هدر في دوائر تنمية وإدارة الموارد البشرية في قطاع التعليم بوزارة التربية والتعليم بسلطنة عمان، حيث

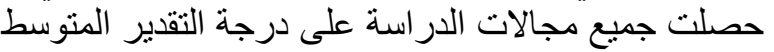
عدم وجود فروق ذات دلالة إحصائية عند مستوى دلالة ( 1 ( 0.05 هفي مستوى الهدر تعزى لمتغير النوع الاجتماعي في محاور الدر اسة ككل. 


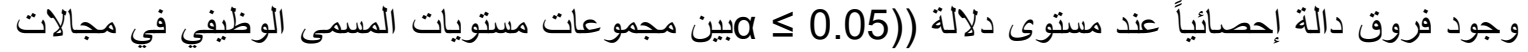
الدراسة ككل في مستوى الهدر في دوائر نتمية وإدارة الموارة المدارد البشرية في قطاع التعليم بوزارة التربية والتعليم بسلطنة عمان.

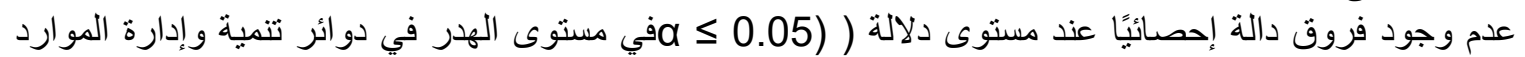

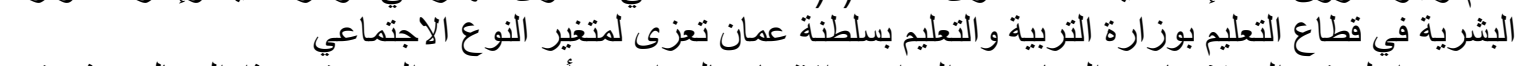

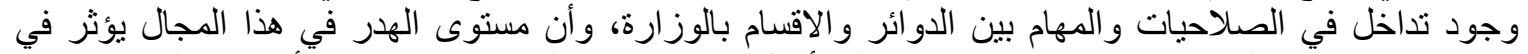

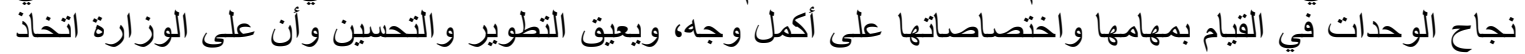

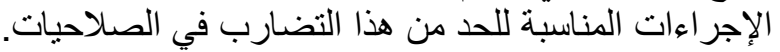

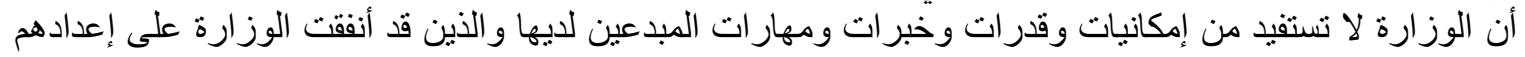

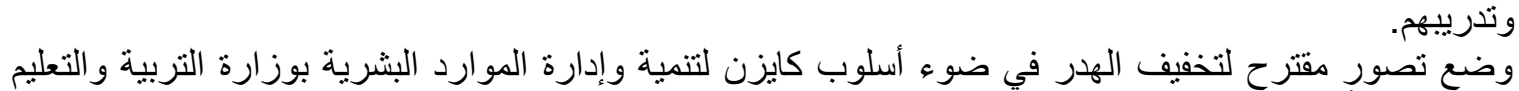
متضمنا عدداً من المتطلبات و المكونات.

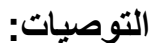
في الختام يوصي الباحث: بما يلي: • تبنى وزارة التربية والتعليم التصور المقترح التي توصلت إليه هذه الدراسة لتطوير الوضع التنظيمي وتخفيف تبنى وزارة التربية والتعليم أسلوب كايزن للتحسين المستمر و القائم على التحسين التدريجي بما يحقق أهداف الوزارة وخططها الخمسية. توفير الدعم اللازم للتحسين المستمر بالمديرات العامة للتربية والتعليم بالمحافظات.

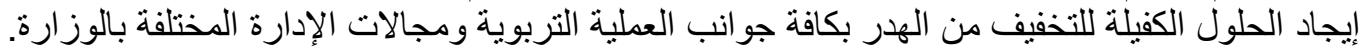

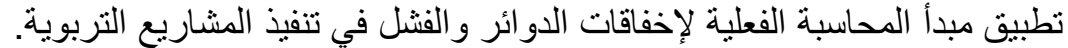

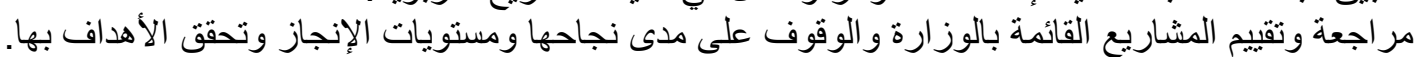

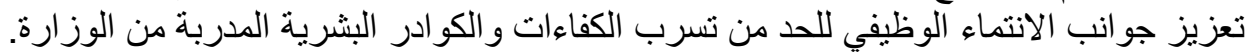

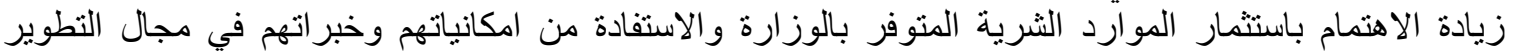

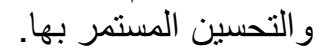
تطوير مسار العمل الإداري من خلال تطبيق استراتيجيات الإدارة الحديثة والتي تتناسب مع رؤية وفلسفة وزارة

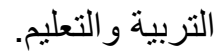
تنفيذ بر امج تدريبية نو عية في مجال التطوير و التحسين للمدر اء ومن في مستو اهم. إيجاد نظام حو افز للإدارة يرتبط بمستوى النجاح وما يحقق من الهداف بالدو ائر.

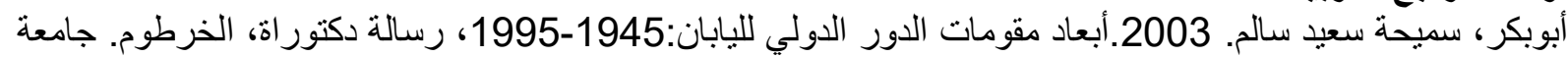
الخرطوم كلية الدراسات الاقتصادية والاجنماعية.

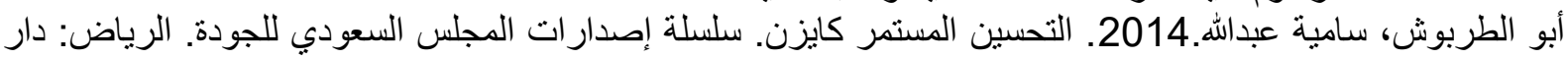
الاصحاب للنشر و التوزيع. تلنه

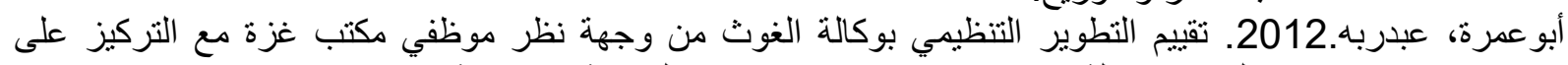

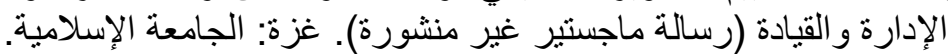

أبوقحف، عبد السلام. 1992.التجربة اليابانية في الإدارة و التنظيم الدعائم الأساسية ومقوماتية الإنة النجاحة، الإسكندرية، الدار

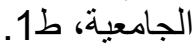

ارحيم، عبد الحميد عبد السلام.2013. الإدارة منهج و أسلوب. الاسكندرية: مؤسسة حورس الدولية للنشر و التوزيع.

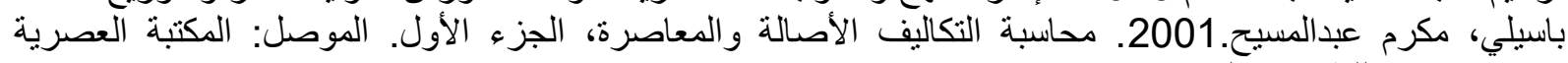
اللطباعة و النشر.

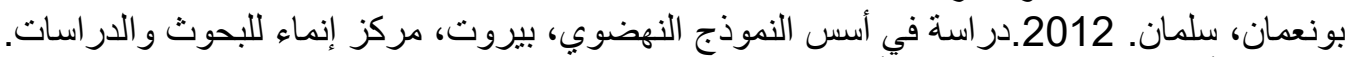

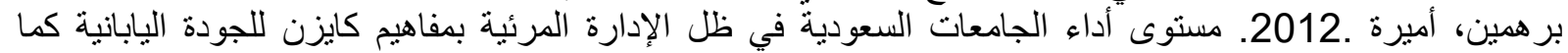

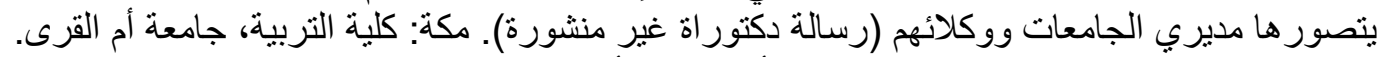

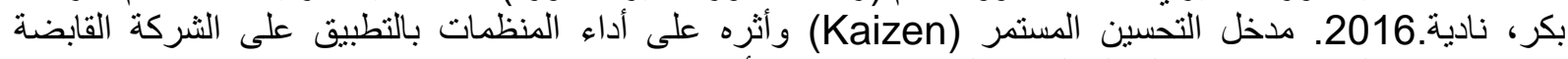

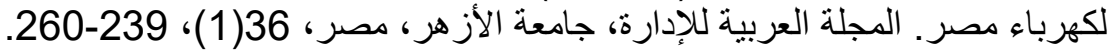




\section{PROVIDING A SCENARIO OF WASTAGE MITIGATION IN THE HUMAN \\ RESOURCE MANAGEMENT SYSTEM BASED ON KAIZEN THEORY IN THE \\ MINISTRY OF EDUCATION OMAN}

البكري، سونيا محمد.2000. إدارة الإنتاج و العمليات مدخل النظم. الإسكندرية: الدار الجامعية.

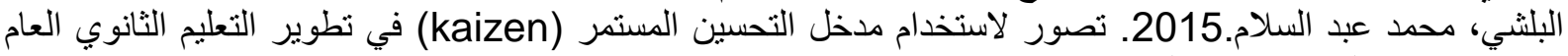

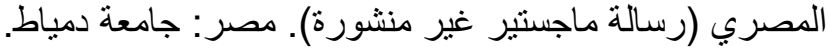

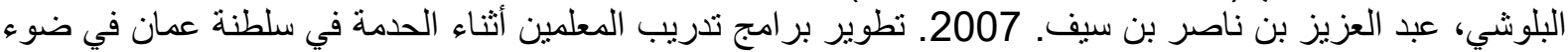

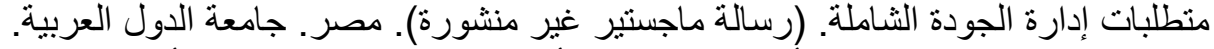

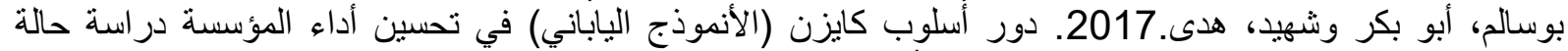

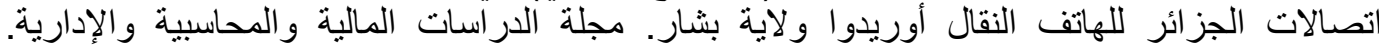

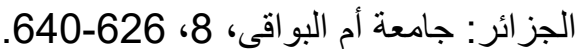

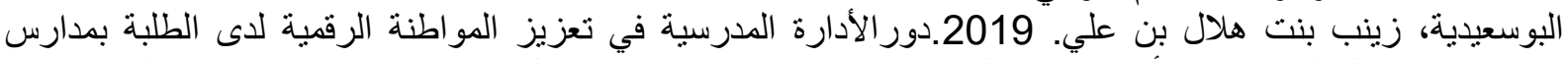
التعليم ما بعد الأساسي في سلطنة عمان (تصور مقترح). (رسالة ماجستير غير منشورة). سلطنة عمان. جامعة السلطان قابوس. كلية التربية التربية.

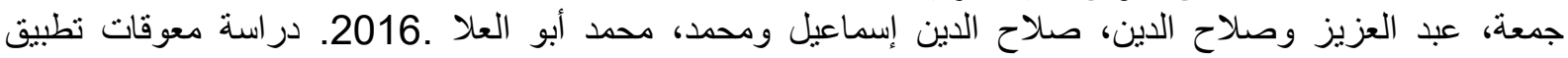
إستراتيجية التحسين المستمر (الكايزن) لتطوير جودة الخدمات الصحية. مصر: المجلة العلمية للإقتصاد

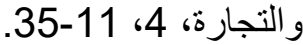
الجهوري، راشد بن حمد بن علي. 2015.تطوير سياسات تنمية الموارد البشرية بوزارة التربية و التعليم في سلطنة عمان

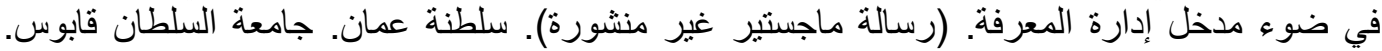
كلية التربية.

جودة، محفوظ أحمد. 2009. إدارة الجودة الثاملة مفاهيم وتطبيقات، عمان، دار و ائل للنشر و التوزيع.

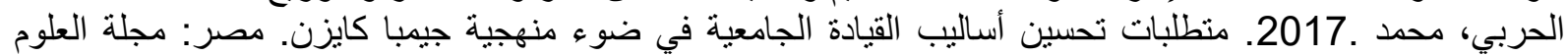
التربوية، 25(1)، 234-232.

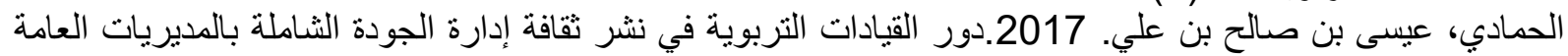

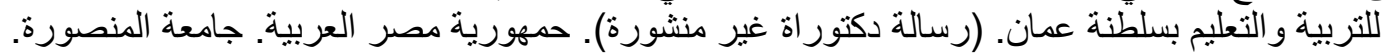

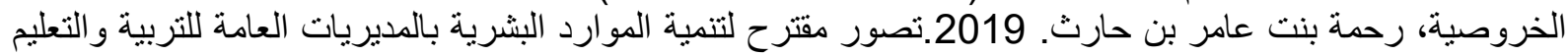
بسلطنة عمان في ضو ضو مدخل الجودة الإحصائي (six sigma). (رسالة ماجستير غير منشورة). سلطنة عمان. جامعة نزوى. كلية العلوم و الآداب.

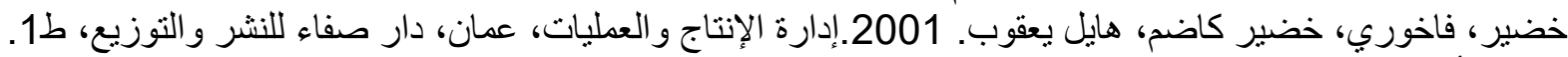
خيري، أسامة. فاخدي، 2013. الإدارة العامة. عمان: دارئ دار الر اية للنشر و التوزيع.

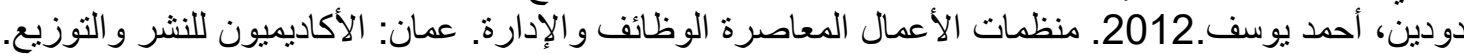

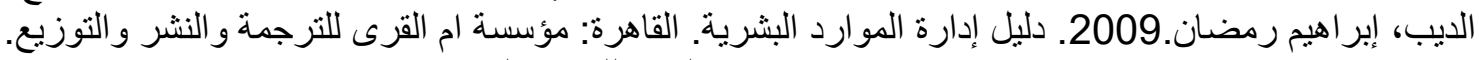
رضا، هاشم حمدي.2011. الإصلاح الإداري.

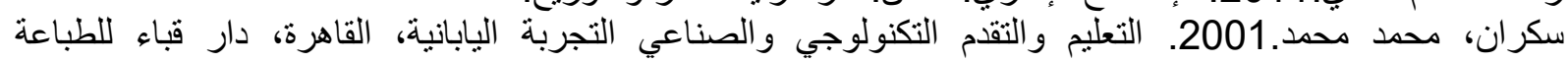

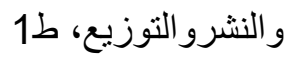

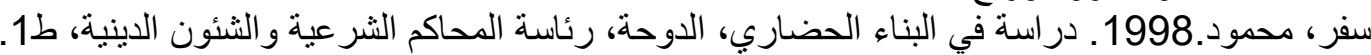

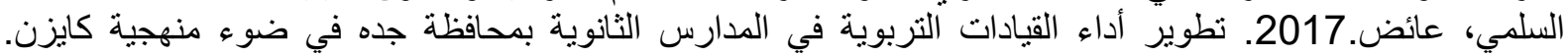

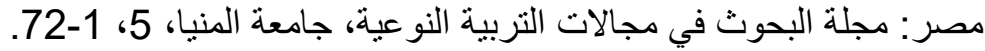

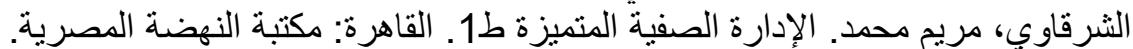

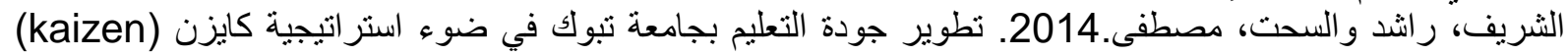

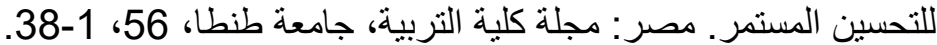

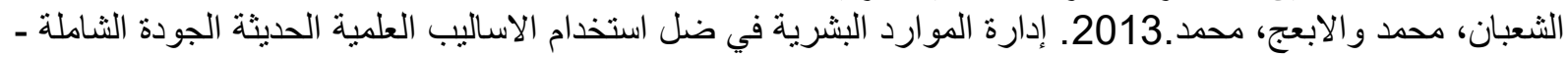
الهندرة. عمان: الرضوان اللنشر و التوزئ.

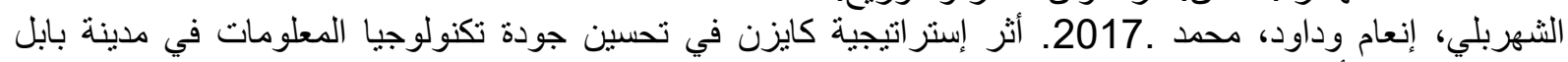

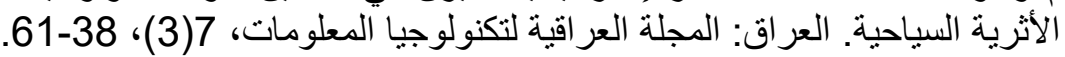

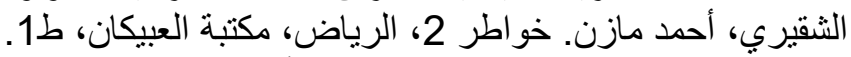

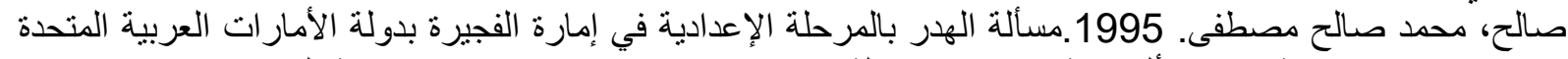

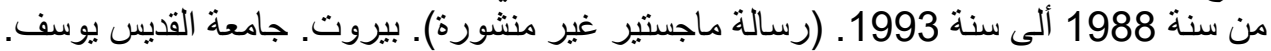

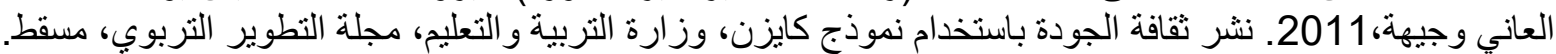


عباس، أنس عبد الباسط ـ2011. إدارة الموارد البشرية. عمان: دار المسيرة للنشر و التوزيع و الطباعة. عبد المحسن، مير غني عبداله، 2010.أثر الهيكل التنظيمي على أداء الثئه المؤسسات، رسالة ماجستير، القاهرة، كلية الدر اسات العليا.

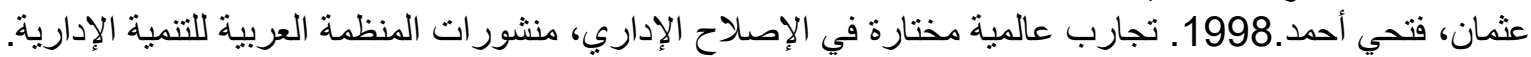

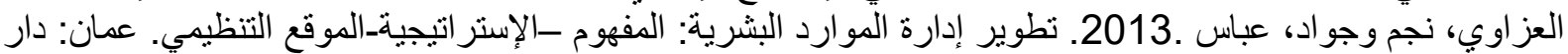
اليازوري العلمية للنشر و التوزيع. العشيري، هشام أحمد يوسف. وزئ. 1997. ظاهرة التسرب في المرحلة الإبتدائية لدولة البحرين. مجلة المعلومات التربوية. البحرين. وزارة التربية و التعليم.

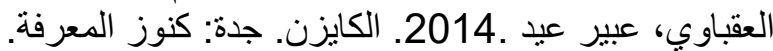
العقدة، صالح و الجو هر، كريمه و أبو سردانة، جمال .2010. دونة دور التدقيق الداخلي في التحسين المستمر للأداء الاجتماعي

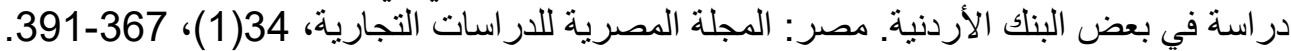

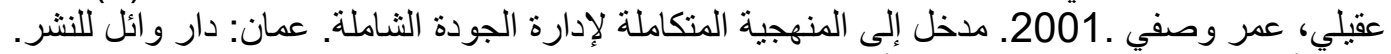

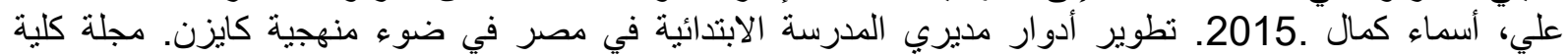

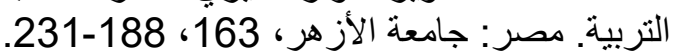

علي، نور، النعيم حسن محمد، أروى عبدالحميدة، مصحمد.2008. إدارة الجودة الثناملة، الخرطوم، هيئة الخرطوم للصحافة

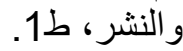

العمري، هاني. 2007. التطوير التنظيمي و إعادة التنظيم، عمان، دار عمرو للنشر و التوزيع.

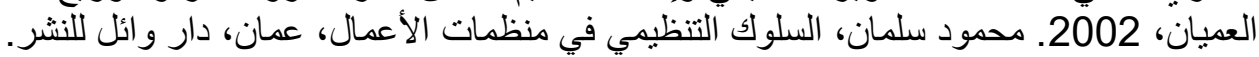

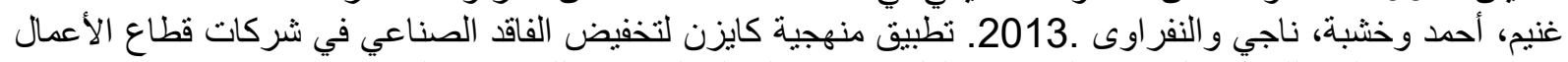

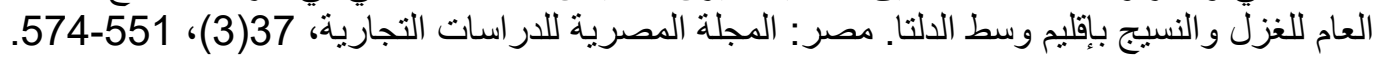

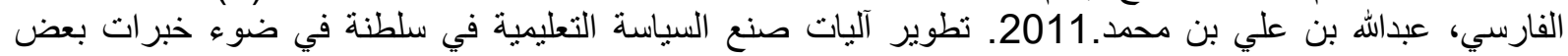

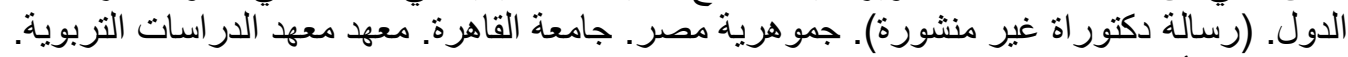

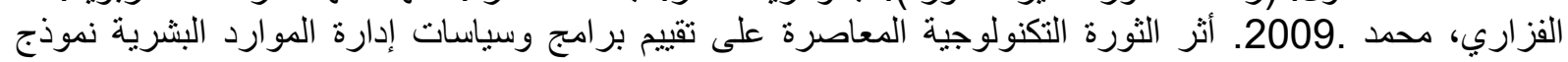

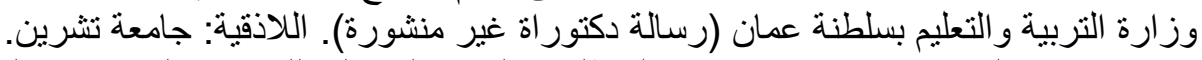

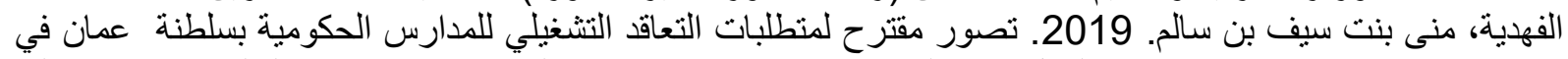

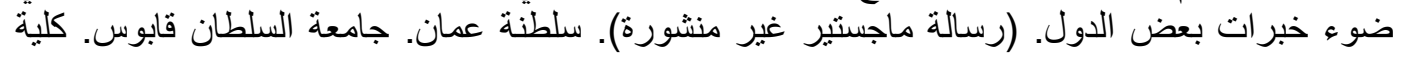
التربية.

فياض، محمود وقدادة، عيسى و عليان، ربحي .2010. مبادئ الإدارة 2: وظائف المنظمة. عمان: دار صفاء للنشر والتوزيع.

القحطاني، عبد المحسن و الرشيدي، نوف والمسعودي، شيماء والعمبري، شيخة .2012. مدى تطبيق مبادئ كايزن

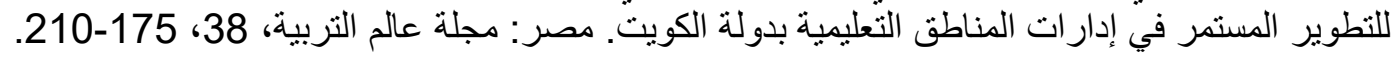

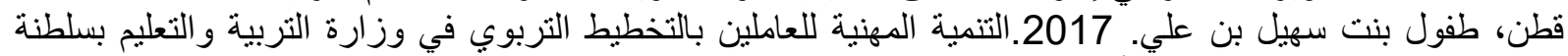

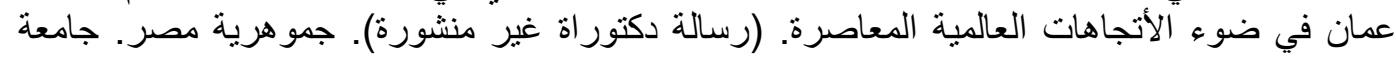
القاهرة. كلية الدر اسات العليا للتربية.

كاظم، حاتم .2011. التحسين المستمر بأسلوب كلفة كانية كايزن و إمكانية اعتماده في الثركة العامة لصناعة الإطارات في

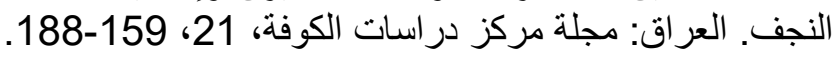

كافي، مصطفى يوسف ـ2014. إدارة الموارد البشرية من منظور إداري -تنموي -تكنولوجي - عولمي. عمان: مكتبة المجتمع العربي للنشر و التوزيع.

كاملية، أمال، بن شلوية، شرفي. 2013.الجودة كمدخل لتحسين الأداء الإنتاجي في المؤسسات العمومية. (رسالة

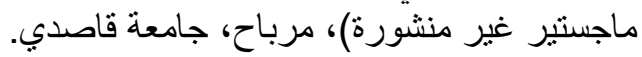

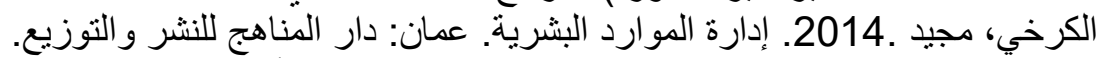

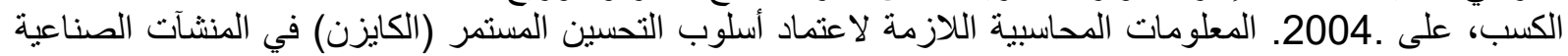

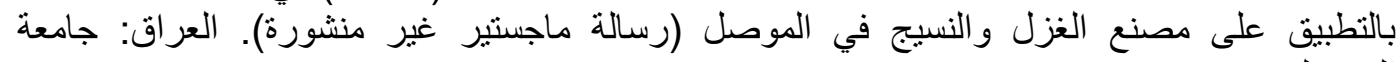
الموصل.

الكسر، شريفة .2018. نظرية كايزن وإمكانية تطبيقها في كلية التربية للبنات بشقراء بالمملكة العربية السعودية من الإنداء

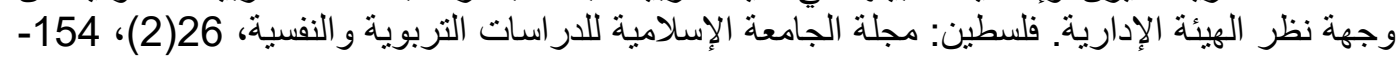

187

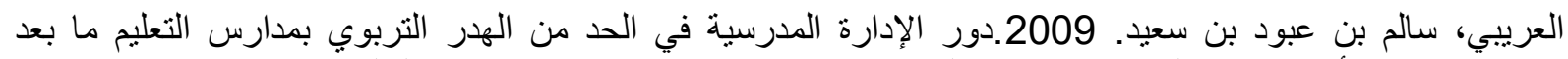
الأساسي في سلطنة عمان. (رسالة ماجستير غير منشورة). مسقط. جامعة السنة السلطان قابوس. 
العويسي، رجب بن علي بن عبيد. 2002. دراسة تقويمية للدور التخطيطي لمدير المدرسة الثنانوية بسلطنة عمان. (رسالة

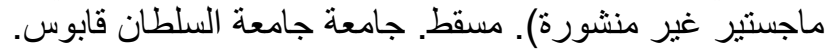

غنيم، أحمد محمد. 2009.المدخل الياباني للتحسين المستمر ومدى استفادة المنظمات العربية، القاهرة، مكتبة العصرية اللنشر و التوزيع.

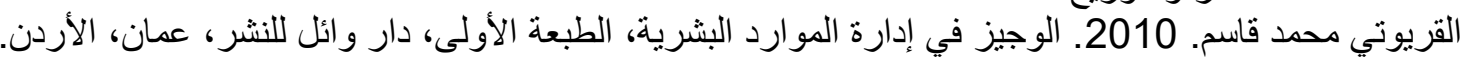

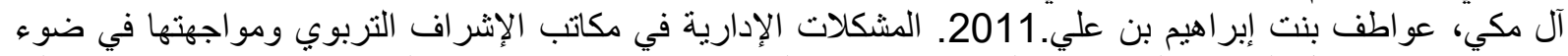
مدخل التطوير التنظيمي بسلطنة عمان. (رسالة ماجستير غير منشورة). سلطنة عمان. جامعة نزوى الإني كلية التية

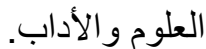

محمد، على .2013. أثر استخدام نموذج كايزن على تنمية مفاهيم التسويق الأخضر و الوعي البيئي لدى طلاب التعليم

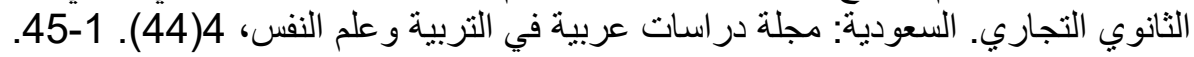

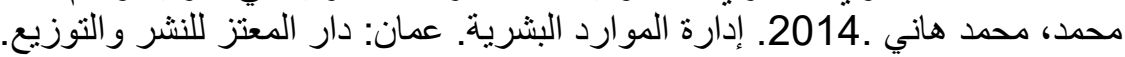

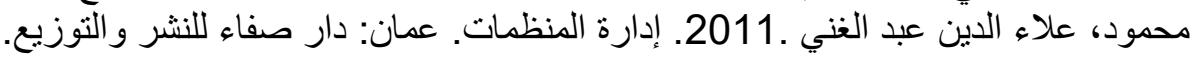

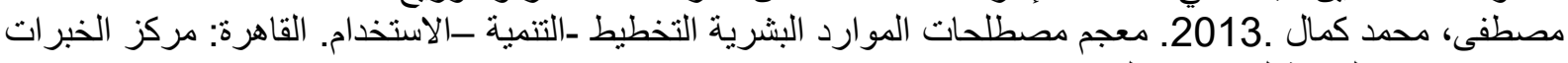
المهنية للإدارة-بميك.

المقبالي، حرشان بن خميس بن حرشان. 2017.تصور مقتر ح لتنمية الموارد البشرية بوزارة التربية وارية التعليم في سلطنة عمان على ضوء منطلبات مجتمع المعرفة. (رسالة دكنوراة غير منشورة). حمهورية مصر التمر العربية.

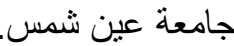

المنيف، إبر اهيم عبدالله. 1998.استر اتيجية الإدارة اليابانية، الرياض، مكتبة العبيكان، ط1.

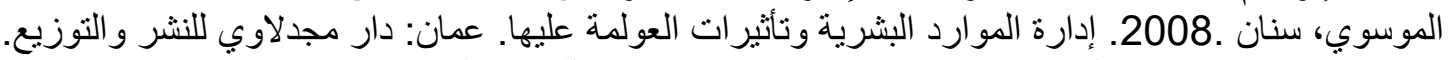

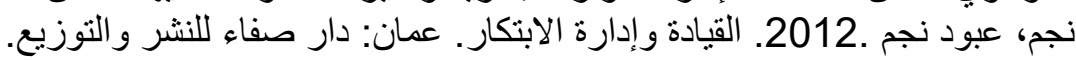

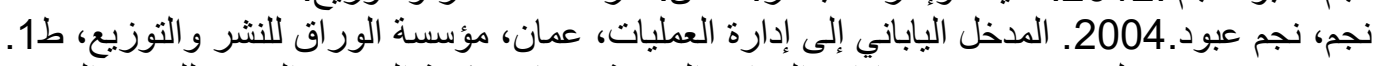

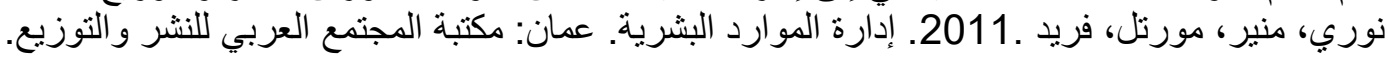

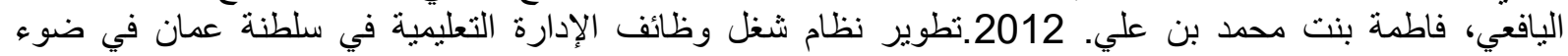

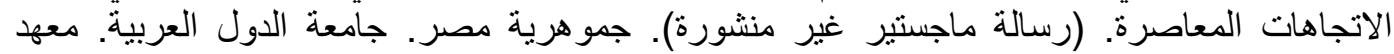
البحوث و الدر اسات العربية.

يوسف، داليا 2013. كيفية استخدام أسلوب جيمبا كايزن في تطوير التعليم الجامعي المصري. 35رئ السعودية: مجلة دراسات عربية في التربية و علم النفس، 35، 351-368.

ثانياً: المراجع الأجنبية

Abbad,M.(1983). Educationai Wastage and its effect on soio-economis and man power provision in algeria.

Bernes,R.G.(1990).school-Based Management at the K-6 Level Overcoming Blockages to Implementation.(Seattle University).

Girardi,Z.(1993). Dropout among secondary school students in Brazil :an exercise in participatory research.

Haslinda, A. \& Hiko, O. (2010) Modeling HRD Practices in Malaysian Manufacturing Firms, Journal of International Social Research.

Powell,T.(2010). Total quality management as competitive advantage: A review and empirical study, Total Quality Management

Sarnovics, a. (2010) Human Resource Development in Organizations of Public Administration of Latvia: A Strategic Approach, Economic and Management.

Sheehan,M.(2014).Human resource management and performance: Evidence from small and medium-sized firms, International Small Business Journal 2014, Vol. 32(5) $545-570$ 
Shickler, R.H (1984). Secondary Management school Problems and solutions : Perceptions for central office Administrations.(University of southern California)

Suárez- Barraza, M,. Ramis- Pujol, J,.\& Sándoval- Arzaga,F.(2011).Finding kaizen approach in small Mexican family businesses: an exploratory study", Journal of Family Business Management, Vol. 1 Iss: 2, pp.107 - 129

Suárez- Barraza,M., \& Ramis- Pujol,J.(2010) "Implementation of Lean- Kaizen in the human resource service process: A case study in a Mexican public service organisation", Journal of Manufacturing Technology Management, Vol. 21

Volume 6 , Issue 5 Iss: 3 , pp.388 - 410

William, S.M. ( 1987 ) . Teacher's attitude to wards, disruptive, behavior in secondary schools.

ثنافثا: شبكة المعلومات الداولية.

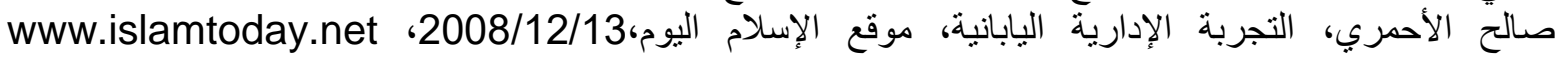

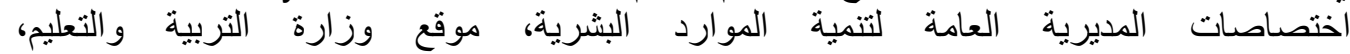
http://www.moe.gov.om/portal

علي جبران، الهدر وارتفاع النفقات وضعف الإنتاجية في الحكومة، موقع الدكتور علي جبران،2013/6/17، www.alijubran.net

فلسفة التعليم في سلطنة عمان، في إنسات

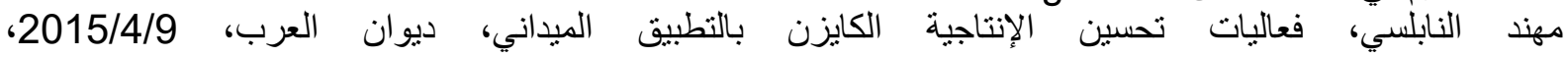
www.diwanalarab.com

https://walikotasurabay.web.app/krejcie-dan-morgan-1970 